\title{
Special issue: Membrane proteins-at the forefront of biophysical research
}

\author{
EBSA Satellite meeting: Structure, Function, Folding and Assembly of Membrane \\ Proteins-Insight from Biophysics, Tata, Hungary, August 2011
}

\author{
Tibor Páli • Balázs Szalontai
}

Received: 25 January 2013/Accepted: 6 February 2013/Published online: 17 February 2013

(c) European Biophysical Societies' Association 2013

Membrane proteins represent very important, complex and challenging research objects. They play essential roles in all life processes of the cellular world, e.g., energisation, transport, catalysis, cellular sensing, communication and mechanics. The host lipid matrix presents an amphiphilic environment for both integral and peripheral membrane proteins. On the one hand, this reduces the conformational space of membrane proteins. On the other hand, the amphiphilic nature of membrane proteins makes them extremely challenging to study outside their native lipid environment. A glance at the sharp contrast between the high abundance of membrane proteins in known genomes and their very limited representation in the protein structure database gives a good sense of the challenges involved. Biophysical approaches to membrane proteins address their purification, reconstitution, prediction and experimental determination of their structure, function, dynamics and folding, physical and molecular modelling, as well as their interactions with each other and the host environment.

The Satellite Conference "Structure, Function, Folding and Assembly of Membrane Proteins-Insight from Biophysics" was held in Tata (Hungary) from August 27 to 31, 2011, as a direct continuation of the 8th European Biophysics Congress, but with themes centred around membrane proteins. It was organised jointly by the following institutions: the Biophysics Workgroup of the Szeged Committee of the Hungarian Academy of Sciences; the Biomembrane Structure Unit (Oxford, UK); the Membrane Section of the Hungarian Biophysical Society; and the

T. Páli $(\bowtie) \cdot$ B. Szalontai

Institute of Biophysics, Biological Research Centre,

Temesvári krt. 62, 6726 Szeged, Hungary

e-mail: tpali@brc.hu
Institute of Biophysics, Biological Research Centre Szeged (Hungary). The idea for the meeting came from Tony Watts, who was a permanent source of tremendous support and help for the local organisers throughout preparation and organisation. Most of the financial support was provided by EBSA, with additional support from the Life from Light Foundation (Hungary) and industrial partners. The conference covered most of the above topics in greater detail than it would have been possible in any session of the mother EBSA Congress, and encompassed 19 talks, about $40 \mathrm{~min}$ each, from experienced researchers. There was plenty of time for discussions in a friendly, relaxing environment (Fig. 1). The meeting took take place in the small historic town Tata, near Budapest, in a hotel situated in a forest on the side of the Old Lake of Tata. Such an environment, where all lectures are held in a single hall, is very refreshing in the era when most large-scale events, including the mother congress, are managed by professional meeting organisers, held in big centres or several buildings, and employ multiple parallel sessions. In this meeting, we organised everything ourselves down to the smallest details, such as having Balázs Szalontai acting as a tourist guide to his hometown during a sight-seeing trip with a restored veteran bus, or enjoying Tibor Páli giving a concert with his band. Another goal of the meeting was to attract researchers early in their scientific careers, offering student fellowships, and letting young researchers learn about key techniques and hot topics, thus enhancing their training in biophysics. In addition, thirteen motivated young researchers could present their project in focus, in the form of shorter talks, and receive advice from other participants and key speakers about their projects. Altogether, we had 70 participants from Europe, India, South America and the US. In this special issue, speakers of the satellite conference and their colleagues present nine 
Fig. 1 Conference photo taken during the lunch break on the first full day of the Satellite Conference (Sunday, September 28, 2011). The organisers: Tibor Páli, host chairman of the conference (1st row, 1st from the right), Balázs Szalontai (1st row, 3rd from the right), Anthony Watts (3rd row, 2nd from the left) and Alajos Bérczi (2nd row, 2nd from the left). (We thank Antoinette Killian for the photo.)

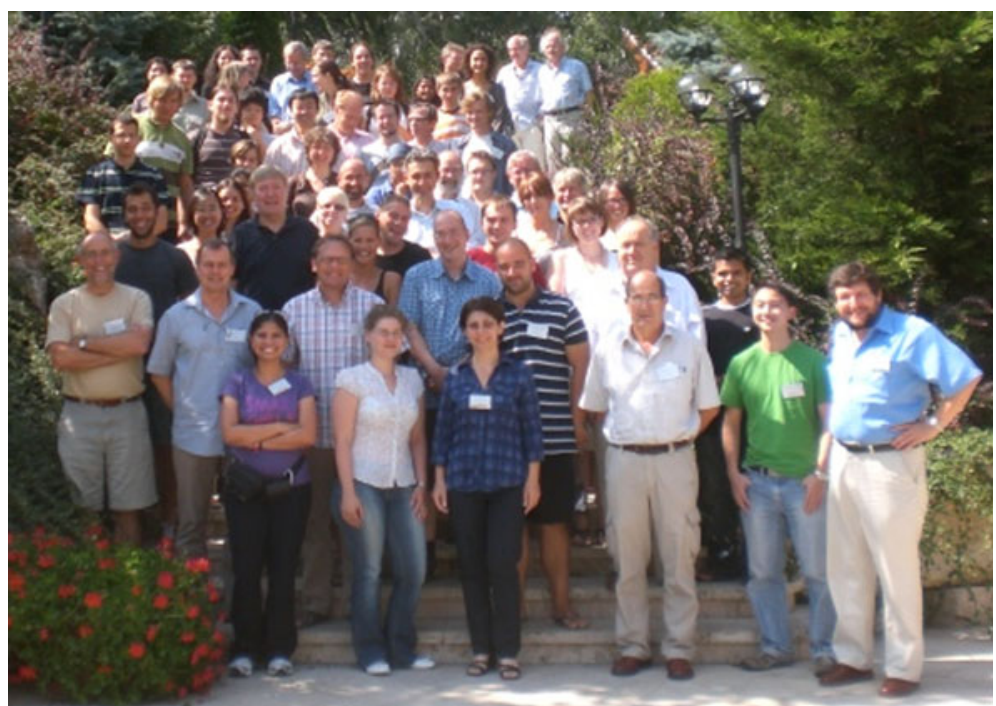

papers. These papers are representative of the following themes: membrane proteins and their environment; membrane transport and channels; advanced structural biology techniques for membrane proteins; and folding and assembly of proteins in membranes.

Whereas in his talk, Tim A. Cross presented evidence from high-resolution solid-state nuclear magnetic resonance spectroscopy that the lipid environment is essential for proper structure of the trans-membrane domain of several membrane proteins, Jean-Luc Popot demonstrated that some other membrane proteins can correctly fold in the presence of amphipols, which are short amphipathic polymers designed to stabilise membrane proteins in aqueous solutions in the absence of detergent. Our special issue starts with a paper (Dahmane et al.), which demonstrates that a membrane or membrane-mimetic environment is not required for correct decoding of the chemical information contained in the sequence of bacteriorhodopsin, and functional folding is possible even in the highly foreign environment of lipid-free amphipols. The paper by Pocanschi et al., in which they studied the folding kinetics of the outer membrane protein A from Escherichia coli, goes on to show that the denatured beta-barrel membrane protein can be folded by dilution of the denaturant urea in the presence of amphipols. On the other hand, weakly bound first-shell or annular lipids surrounding membrane proteins, studied extensively with spin label electron paramagnetic resonance spectroscopy, have been shown in the past to be of functional relevance. In their contribution, Marsh and Páli studied a much less well-known class of lipids, namely those that are strongly bound to membrane proteins. Their paper presents an extensive analysis of the conformation of all lipids found in the PDB structures of membrane proteins. They itemise and illustrate the necessary features of conformational and stereochemical restraints needed to avoid stereochemical violations of lipids in X-ray structures. It should be noted that many of the embedded lipids have been shown earlier to be essential for the functioning of the respective membrane protein. The above apparent contradiction over the issue of the role of the environment of membrane protein structure and function was the source of some interesting discussions and some less serious exchanges of arguments (Fig. 2) during the meeting.
Fig. 2 Jean-Luc Popot (left) and Tim A. Cross (right) engaged in a friendly exchange of arguments about the role of the hosting environment in membrane protein folding and function. The photo was taken (by Arunima Chaudhuri, one of the student participants) right before the dinner followed by wine tasting, one of the social events of the conference

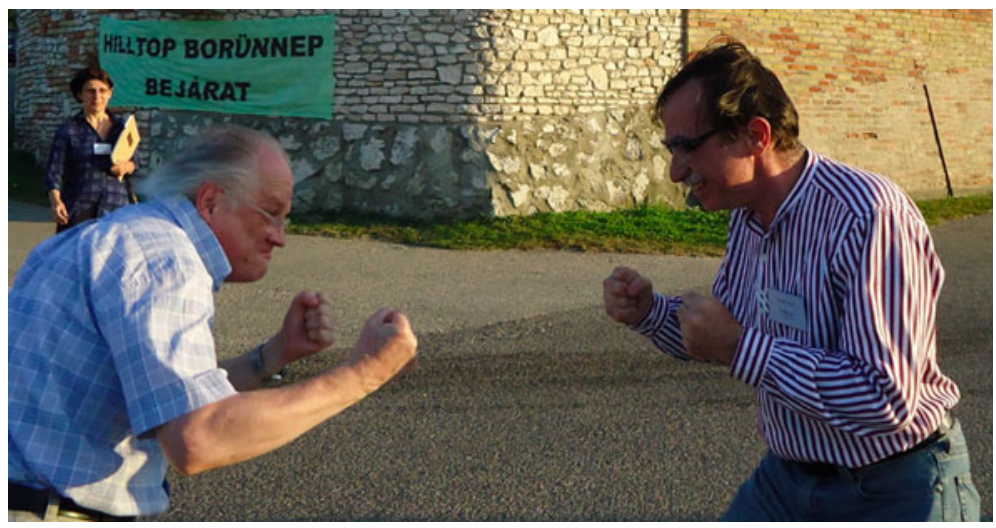


Three papers are related to the topic of membrane transport. Ferencz et al. report the rate of rotation of the rotor in the yeast vacuolar proton-ATPase, a membranebound proton pump and molecular rotary engine, for the first time in native membranes, under standardised conditions. Bérczi et al. present spectroscopic data demonstrating that the tonoplast-localised and the putative tumour suppressor cytochrome b561 proteins can be reduced by reductants other than ascorbate and dithionite. The cytochrome b561 proteins constitute a family of trans-membrane di-heme proteins that are present in a wide variety of organisms. They have two distinct b-type heme groups localised between four trans-membrane helices and are close to the two opposite sides of the membrane. This conserved architecture functions as a trans-membrane electron channel in these proteins. Erdei et al. studied a plasma membrane multidrug transporter (ABCG2), which has an established role in the drug-resistance of cancer cells. Their paper is about the heterogeneity of the expression pattern of ABCG2 in human embryonic stem cell lines, and whether this heterogeneity is related to stress responses. They conclude that a dynamic balance of ABCG2 expression at the population level has the advantage of enabling prompt response to changes in the cellular environment.

Among novel techniques targeting membrane proteins, site-directed spin labelling and pulsed electron paramagnetic resonance spectroscopy combined with molecular modelling is a very powerful approach, as demonstrated by the paper of Gunnar Jeschke. He presents an analysis of the known high-resolution protein structures with the LeuT fold, a core of two five-helix inverted repeats, by structural superposition of the core trans-membrane domains. The data are directly comparable with the structural and dynamic information that can be obtained with the above approach, especially the static and even dynamic distance distributions of spin-labelled amino acids. The results support the rocking bundle model for the major conformational change between the outward- and inward-facing states of these proteins, and point to an important role for the independently moving last trans-membrane domains of each repeat in occluding access to the central binding site. The paper by Baeza-Delgado et al. is also based on experimental structures, since a large-scale analysis of the prevalent locations of amino acids of 170 structures of integral membrane proteins and 930 structures of water-soluble helical proteins is presented. The distribution of amino acids along trans-membrane helices is also examined. Their results obviously aid membrane protein structure prediction algorithms, and should also prove useful for constructing models of membrane proteins with desired properties.

Our special issue concludes with a review on the structure-function correlations of pulmonary surfactant protein SP-B and the saposin-like family of proteins (Olmeda et al.). Pulmonary surfactant is a lipid-protein complex secreted by the respiratory epithelium of mammalian lungs, which plays an essential role in stabilising the alveolar surface and so reducing the work of breathing. The paper summarises the most relevant features of the structure, lipid-protein and protein-protein interactions of SP-B and the saposin-like family of proteins, as a basis to propose an integrated model and a common mechanistic framework of the apparent functional versatility of the saposin fold. 